\title{
Strong Correlation to Weak Correlation Phase Transition in Bilayer Quantum Hall Systems
}

\author{
John Schliemann, S. M. Girvin, and A. H. MacDonald \\ Department of Physics, Indiana University, Bloomington, Indiana 47405-7105
}

(Received 20 June 2000)

\begin{abstract}
At small layer separations, the ground state of a $\nu=1$ bilayer quantum Hall system exhibits spontaneous interlayer phase coherence. The evolution of this state with increasing layer separation $d$ has been a matter of controversy. We report on small system exact diagonalization calculations which suggest that a single-phase transition, likely of first order, separates incompressible states with strong interlayer correlations from compressible states with weak interlayer correlations. We find a dependence of the phase boundary on $d$ and interlayer tunneling amplitude that is in very good agreement with recent experiments.
\end{abstract}

DOI: 10.1103/PhysRevLett.86.1849

The ground state of a two-dimensional monolayer electron system at Landau level filling factor $\nu=1$ is a single Slater determinant described exactly by Hartree-Fock theory and is a strong ferromagnet with a large gap $E_{g}$ for charged excitations $[1,2]$. This elementary property has rich and interesting consequences for the physics of bilayer quantum Hall systems at the same total $\nu$, consequences that are readily appreciated when a pseudospin language $[1,3]$ is used to describe the layer degree of freedom. When the layer separation $d$ goes to zero, interactions between layers are identical to interactions within layers. The pseudospin bilayer Hamiltonian is then identical to the single layer Hamiltonian with spin and its ground state has pseudospin order and a finite charge gap. For infinite layer separation, on the other hand, the bilayer system reduces to two disordered, compressible, uncorrelated $\nu=1 / 2$ systems. This Letter concerns the evolution of bilayer quantum Hall systems between these two extremes.

For small layer separations the difference between interlayer and intralayer interactions breaks the pseudospin invariance of the Hamiltonian, resulting in an incompressible easy-plane pseudospin ferromagnet. In physical terms the pseudospin order represents spontaneous phase coherence between the electron layers. Several scenarios have been proposed for the evolution of the ground state as the layer separation increases further. In Hartree-Fock theory [4], spontaneous interlayer coherence is lost if the layer separation exceeds a critical value, and the ground state at large separations consists of weakly correlated Wigner crystals. While possibly instructive, this picture is known to be incorrect at large $d$ since half-filled Landau levels do not have crystalline ground states. Working in the other direction, Bonesteel et al. started [5] from the composite fermion theory of isolated compressible $\nu=1 / 2$ layers, and concluded that coupling would lead to pairing between composite fermions in opposite layers and also, implicitly, to a charge gap. Since the pseudospin ferromagnet possesses particle-hole rather than particle-particle pairing, however, this picture still implies that at least one phase transition occurs as a function of layer separation. In a numerical diagonalization study $\mathrm{He}$
PACS numbers: 73.43.-f, 73.21.-b

et al. [6] predicted, on the basis of the system parameter dependence of overlaps between exact ground states and two different variational wave functions, the existence of two distinct incompressible states separated by a region of compressible states.

Experiments, on the other hand, have tended to be consistent [7] with the proposal [3] that a single-phase transition from an incompressible to a compressible state occurs with increasing layer separation at any value of the interlayer tunneling amplitude. Very recently, in an intriguing new experiment by Spielman et al. [8], the tunneling conductance across the layers was studied in a sample with extremely small tunneling amplitude. When the ratio of layer separation and magnetic length was lowered (at fixed filling factor) below a critical value, the conductance showed a very pronounced peak around zero bias voltage between the layers that provides direct evidence [9] for spontaneous interlayer phase coherence. This is because in the coherent state the layer index is uncertain. Only in this case can tunneling leave the system in or near its ground state, avoiding the orthogonality catastrophe and allowing tunneling to occur near zero voltage.

Since the critical layer separation found by Spielman et al. is close to the one obtained earlier by Murphy et al. for the onset of the quantum Hall effect [7], experiment demonstrates that for vanishing tunneling amplitude the phase transitions at which pseudospin order and the charge gap are lost are either closely spaced or coincident.

In this Letter we report on small system exact diagonalization calculations which strongly suggest that bilayer quantum Hall systems have a single-phase transition, likely of first order, as a function of $d$. Our critical layer separation is in very good quantitative agreement with the value measured in Ref. [8]. In the light of the experimental results mentioned above, our calculations imply that the charge gap disappears and long-range phase coherence simultaneously drops sharply to near zero at the phase transition. This result is not entirely unexpected since a simple Landau-Ginzburg analysis indicates that the two order parameters could not vanish simultaneously without fine-tuning, if the transition were continuous. Also the 
mean-field theory energy gap is proportional to the pseudospin order parameter, suggesting that these two orders are mutually reinforcing and that a first order transition is therefore likely. Finally, we note that, experimentally, the charge gap phase transition is sharp even at finite tunneling between the layers. Since tunneling produces a pseudomagnetic field which couples to the pseudospin order parameter, this is an unusual magnetic transition which does not involve symmetry breaking, a fact which lends further weight to the suggestion that the transition is first order.

We analyze bilayer quantum Hall systems numerically by means of exact diagonalizations of finite systems using the spherical geometry. We have verified numerically that the ground state and low-lying excitations are fully spin polarized and neglect the spin degree of freedom in the present discussion. The Hamiltonian is given by

$$
\mathcal{H}=\mathcal{H}_{1 \mathrm{P}}+\mathcal{H}_{\text {Coul }},
$$

where $\mathcal{H}_{\text {Coul }}$ represents the usual Coulomb interaction within and between layers, and the single-particle Hamiltonian $\mathcal{H}_{1 \mathrm{P}}$ is given by

$$
\mathcal{H}_{1 \mathrm{P}}=-\frac{1}{2} \sum_{m} c_{\mu, m}^{+}\left[\Delta_{v} \tau_{\mu, \mu^{\prime}}^{z}+\Delta_{t} \tau_{\mu, \mu^{\prime}}^{x}\right] c_{\mu^{\prime}, m} .
$$

We concentrate here on the tunneling amplitude $\left(\Delta_{t}\right)$ tuned phase transition, although bias voltage $\left(\Delta_{v}\right)$ dependence is also interesting and often experimentally more convenient. $\mu, \mu^{\prime} \in\{+,-\}$ run over the layer (or pseudospin) indices and a summation convention is implicit; $\vec{\tau}$ are the pseudospin Pauli matrices. $m \in\left\{-N_{\phi} / 2, \ldots, N_{\phi} / 2\right\}$ is the $z$ projection of the orbital angular momentum of each electron in the lowest Landau level, where $N_{\phi}$ is the number of flux quanta penetrating the sphere. In the following we denote the pseudospin operators by $\vec{T}=$ $(1 / 2) \sum_{m} c_{\mu, m}^{+} \vec{\tau}_{\mu, \mu^{\prime}} c_{\mu^{\prime}, m}$. The interlayer separation $d$ is measured in units of the magnetic length $l_{B}=\sqrt{\hbar c / e B}$, and all energies are given in units of the Coulomb energy scale $e^{2} / \epsilon l_{B}$. We consider the case of zero well width to enable comparison with most previous theoretical investigations [10-13], and also systems consisting of two rectangular wells of finite width $w$ [14] whose ratio to the center-to-center layer separation $d$ is $w / d=0.65$. This value corresponds to the sample used in Ref. [8].

We consider systems with an even electron number $N$ which leads to a nondegenerate spatially homogeneous ground state with total angular momentum $L=0$. For simplicity, let us first examine the case of vanishing bias voltage, where both $\left\langle T^{y}\right\rangle$ and $\left\langle T^{z}\right\rangle$ are strictly zero.

Figure 1 shows the interlayer phase coherence as measured by the expectation value $\left\langle T^{x}\right\rangle$ along with the fluctuation $\Delta T^{x}=\sqrt{\left\langle T^{x^{2}}\right\rangle-\left\langle T^{x}\right\rangle\left\langle T^{x}\right\rangle}$ as a function of the tunneling gap for a system of 12 electrons, a layer separation of $d=1.80$, and zero well width. At $\Delta_{t}=0,\left\langle T^{x}\right\rangle$ is necessarily zero in a finite system. With increasing tunneling gap, $\left\langle T^{x}\right\rangle$ grows rapidly reaching an inflection point

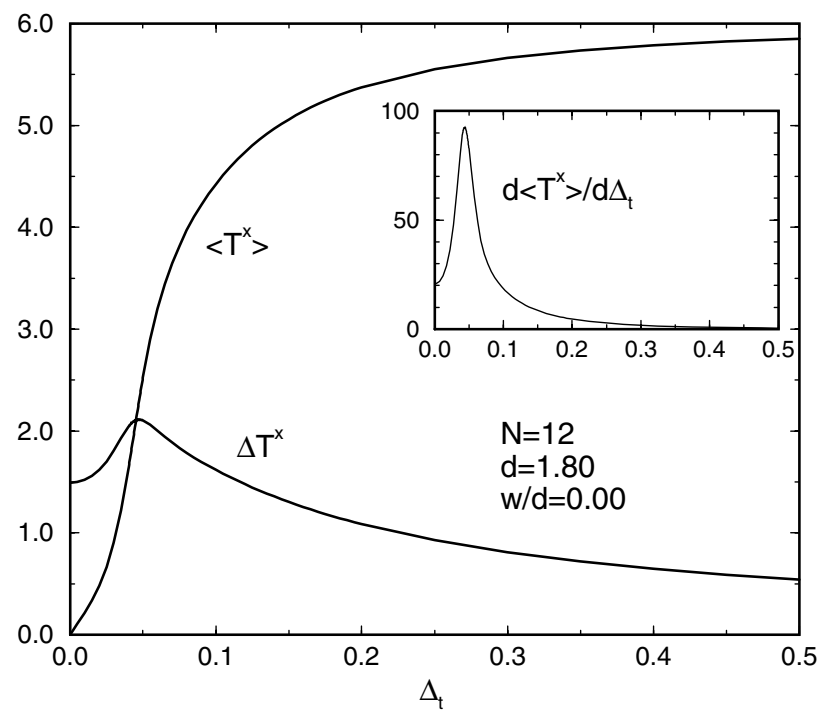

FIG. 1. The pseudospin expectation value $\left\langle T^{x}\right\rangle$ and the fluctuation $\Delta T^{x}$ as a function of the tunneling gap $\Delta_{t}$. The derivative $d\left\langle T^{x}\right\rangle / d \Delta_{t}$ [measured in units of $1 /\left(e^{2} / \epsilon l_{B}\right)$ ] is shown in the inset.

with a very steep tangent. The differential pseudospin susceptibility, $\chi=(1 / N)\left\langle T^{x}\right\rangle / d \Delta_{t}$, is plotted in the inset and shows a very pronounced peak. In the immediate vicinity of this peak, the pseudospin fluctuation $\Delta T^{x}$ has also a pronounced maximum. In Fig. 2 the $\chi$ is plotted for different numbers of electrons.

The rapid growth with increasing system size of the peak in this generically intensive quantity is strong evidence for a ground state phase transition. Analogous findings are obtained for the peak in the pseudospin fluctuation. Thus, the peaks in the susceptibility of the pseudospin and its fluctuation grow very rapidly with increasing system size and signal a quantum phase transition at the critical

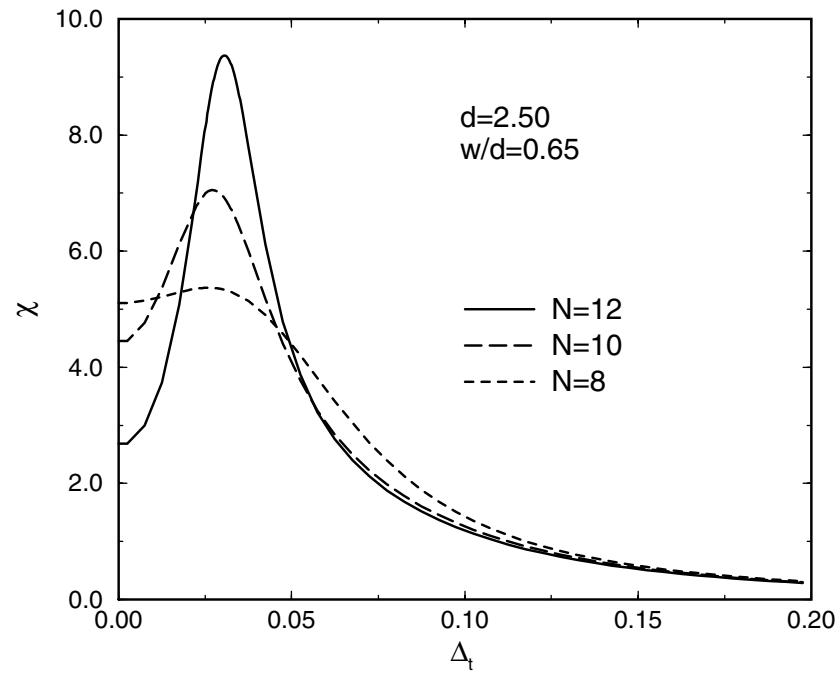

FIG. 2. The pseudospin susceptibility $\chi$ for different system sizes as a function of the tunneling gap. The rapidly growing peak indicates a quantum phase transition. 
value of the tunneling gap. At large tunneling the system pseudospin magnetization is close to its maximum value, while at small (but also finite) tunneling the system is disordered and the pseudospin magnetization is strongly reduced by interactions.

The two peaks described above occur at extremely nearby values of $\Delta_{t}$ at a given layer separation $d$, and we consider the very tiny differences in their location as a finite-size effect. To estimate the phase diagram of the system we place the phase boundary at the maximum of the quantum fluctuations $\Delta T^{x}$.

Figure 3 shows the resulting phase boundaries for different system sizes and both cases of well width. At small layer separation the system is in the ordered phase and the fluctuation peak occurs exactly at $\Delta_{t}=0$. At a critical layer separation $d_{c}\left(\Delta_{t}=0, N\right)$ the phase boundary moves out rapidly to finite values of $\Delta_{t}$ and intersects the axis $\Delta_{t}=0$ with an almost horizontal tangent. This is in qualitative agreement with earlier experimental [7] and theoretical [3] estimates of the phase diagram.

The critical values $d_{c}\left(\Delta_{t}=0, N\right)$ form a rapidly converging data sequence and are plotted in Fig. 4. These finite-size data are accurately and consistently described by an ansatz of the form $d_{c}(N)=\alpha+\beta N^{-\lambda}$ with two fit parameters $\alpha=d_{c}(N=\infty), \beta$, and a shift exponent $\lambda$. The best fits to both sets of data are obtained for $\lambda=5.0 \pm 0.2$ leading to a value of $d_{c}(N=\infty)=$ $1.30 \pm 0.03$ for zero well width, and $d_{c}(N=\infty)=$ $1.81 \pm 0.03$ for $w / d=0.65$. The latter value is in excellent agreement with the results of Ref. [8], where the onset of the tunneling conductance peak is observed at a layer separation of $d=1.83$. Thus, our numerical results clearly indicate that the findings of the above tunneling experiments are the signature of a quantum phase transition. The very large value of $\lambda$ seems inconsistent with a diverging correlation length and suggests the transition is first order. A first order phase transition

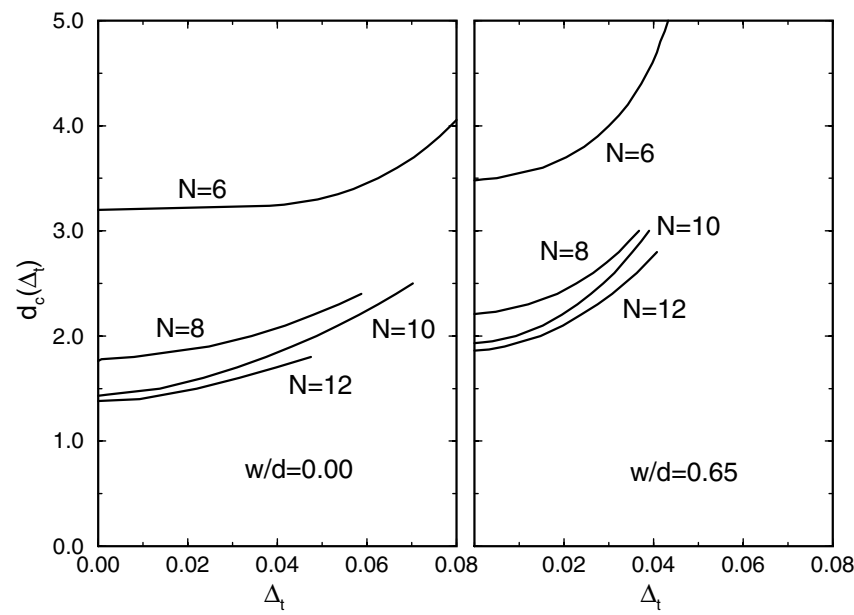

FIG. 3. Phase boundaries for different system sizes $N$ and ratios of well width to layer separation. would explain the apparent coincidence of the appearance of spontaneous phase coherence and the quantum Hall effect in experiment $[7,8]$. We note that our result for the critical layer separation at vanishing tunneling gap agrees reasonably, at zero well width, with the point at which the uniform density phase coherent state first becomes unstable in the Hartree-Fock approximation [3]. At larger $w$, however, the Hartree-Fock estimates clearly deviate from the exact diagonalization result.

In order to further investigate the order of the quantum phase transition, we introduce the ratio

$$
\omega_{N}=\frac{2\left(\Delta T^{x}\right)_{N}^{2}}{\left(d\left\langle T^{x}\right\rangle / d \Delta_{t}\right)_{N}},
$$

where the subscript $N$ refers to the system size. As we discuss below, this type of ratio should prove to be a powerful general tool in the analysis of any quantum phase transition. In classical physics this ratio of fluctuation to susceptibility is equal to the thermal energy $k_{B} T$ and vanishes at $T=0$. The classical relationship does not apply here since the Hamiltonian fails to commute with its derivative with respect to $\Delta_{t}$. There is, however, a closely related zero-temperature relationship with the typical excitation energy $\omega_{N}$ taking over the role of temperature. The fluctuation can be written as

$$
\left(\Delta T^{x}\right)^{2}=\sum_{n>0}\left|\left\langle n\left|T^{x}\right| 0\right\rangle\right|^{2},
$$

where the sum is performed over all excited states, while for the derivative of the pseudospin magnetization one finds from linear response theory

$$
\frac{d\left\langle T^{x}\right\rangle}{d \Delta_{t}}=2 \sum_{n>0} \frac{\left|\left\langle n\left|T^{x}\right| 0\right\rangle\right|^{2}}{E_{n}-E_{0}} .
$$

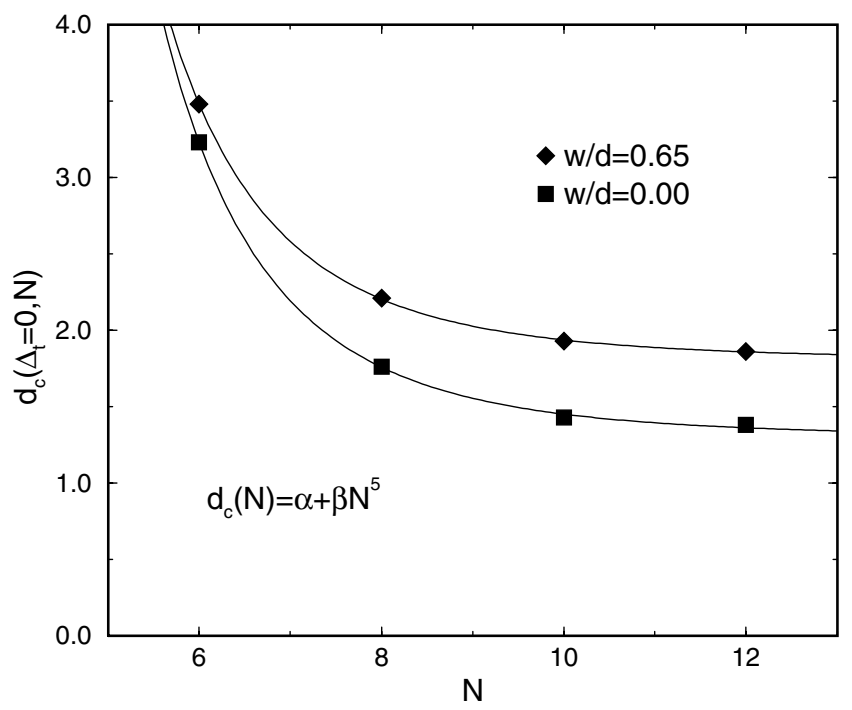

FIG. 4. The critical layer separation $d_{c}\left(\Delta_{t}=0, N\right)$ (filled symbols) at vanishing tunneling as a function of the system size $N$ for both cases of well width. The lines are finite-size fits to the data with a shift exponent of $\lambda=5.0$. 
From these equations we see that $\omega_{N}$ is a harmonic average of excitation energies $\left(E_{n}-E_{0}\right)$, weighted by the factors $\left|\left\langle n\left|T^{x}\right| 0\right\rangle\right|^{2}$. In particular, $\omega_{N}$ has a vanishing thermodynamic limit if at least one state with a nonvanishing matrix element $\left\langle n\left|T^{x}\right| 0\right\rangle$ has an excitation energy $\left(E_{n}-E_{0}\right)$ which extrapolates to zero for $N \rightarrow \infty$. Thus, Eq. (3) defines a characteristic energy scale of the system at the phase boundary. The operator $T^{x}$ naturally enters this expression since it couples to a control parameter driving the phase transition.

For a continuous phase transition one would clearly expect $\omega_{N}$ to vanish at the phase boundary for an infinite system, while a finite $\operatorname{limit} \lim _{N \rightarrow \infty} \omega_{N}$ is indicative of a finite energy scale, i.e., a first order transition. From our finite-size data for $\omega_{N}$ [evaluated at vanishing tunneling and $d=d_{c}(N)$ ] we conclude that this quantity extrapolates for $N \rightarrow \infty$ to a rather substantial nonzero value of order $0.05 e^{2} / \epsilon l_{B} \sim 5 \mathrm{~K}$ for both values of $w$ considered here. Along with the arguments and experimental findings given so far, this result strongly suggests that the bilayer quantum Hall system at filling factor $\nu=1$ undergoes a single first order phase transition as a function of the ratio of layer separation and magnetic length at all values of the tunneling amplitude. The phase boundary separates a phase with strong interlayer correlation (and a finite gap for charged excitations) from a phase with weak interlayer correlations and vanishing $E_{g}$.

Finally we comment briefly on the influence of a bias voltage between the layers. When applying a bias voltage to the system the vector $\langle\vec{T}\rangle$ is tilted out of the $x y$ plane with a finite $z$ component. In this case we find numerically that the quantum phase transition is again signaled by the longitudinal fluctuation of the pseudospin magnetization and its susceptibility, and all results concerning the phase boundary are qualitatively the same. This agrees with experimental results by Sawada et al., who found a remarkable stability of the $\nu=1$ quantum Hall state against a finite bias voltage, as compared to the behavior at other filling factors [15]. First order phase transitions from strongly correlated to weakly correlated states also occur with increasing bias potential. We predict measurable anomalies in the double-layer system capacitance at bias tuned phase transitions.

We acknowledge helpful discussions with S. Sachdev. This work was supported by the Deutsche Forschungsgemeinschaft under Grant No. SCHL 539/1-1 and by the
National Science Foundation under Grants No. DMR9714055 and No. DMR-0087133.

[1] For a review on experimental research see J. P. Eisenstein, in Perspectives in Quantum Hall Effects, edited by S. Das Sarma and A. Pinczuk (Wiley, New York, 1997); for a review on theoretical research see S. M. Girvin and A. H. MacDonald, in the same volume.

[2] B. I. Halperin, Helv. Phys. Acta 56, 75 (1983).

[3] A.H. MacDonald, P.M. Platzman, and G. S. Boebinger, Phys. Rev. Lett. 65, 775 (1990).

[4] R. Côte, L. Brey, and A. H. MacDonald, Phys. Rev. B 46, 10239 (1992).

[5] N. E. Bonesteel, I. A. McDonald, and C. Nayak, Phys. Rev. Lett. 77, 3009 (1996).

[6] S. He, S. Das Sarma, and X. C. Xie, Phys. Rev. B 47, 4394 (1993).

[7] S. Q. Murphy, J. P. Eisenstein, G. S. Boebinger, L. N. Pfeiffer, and K. W. West, Phys. Rev. Lett. 72, 728 (1994).

[8] I. B. Spielman, J. P. Eisenstein, L. N. Pfeiffer, and K. W. West, Phys. Rev. Lett. 84, 5808 (2000).

[9] A. Stern, S. M. Girvin, A. H. MacDonald, and N. Ma, cond-mat/0006457; L. Balents and L. Radzihovsky, cond-mat/0006450; M.M. Fogler and F. Wilczek, cond-mat/0007403; E. Demler, C. Nayak, and S. Das Sarma, cond-mat/0008137.

[10] K. Moon, H. Mori, K. Yang, S. M. Girvin, A. H. MacDonald, L. Zheng, D. Yoshioka, and S.-C. Zhang, Phys. Rev. B 51, 5138 (1995).

[11] K. Yang, K. Moon, L. Belkhir, H. Mori, S. M. Girvin, A. H. MacDonald, L. Zheng, and D. Yoshioka, Phys. Rev. B 54, 11644 (1996).

[12] K. Moon, Phys. Rev. Lett. 78, 3741 (1997).

[13] Y. N. Joglekar and A. H. MacDonald, Physica (Amsterdam) 6E, 371 (2000).

[14] To compute the pseudopotentials of the Coulomb interaction in the case of finite well width we take the electron wave function for the growth direction in each well to be a normalized sine having its zeros at the edges of the well. This means we neglect electron charge density outside the wells, which is a very good approximation for not too large tunneling $\Delta_{t} \lesssim 0.2\left(e^{2} / \epsilon l_{B}\right)$, where the Coulomb energy scale is of order $100 \mathrm{~K}$. An illustrative picture of the charge density distribution can be found in Ref. [6].

[15] A. Sawada, Z. F. Ezawa, H. Ohno, Y. Horikoshi, O. Sugie, S. Kishimoto, F. Matsukura, Y. Ohno, and M. Yasumoto, Solid State Commun. 103, 447 (1997). 